\title{
Narcolepsy Presenting as Refractory Epilepsy
}

Sir,

Narcolepsy refers to a syndrome characterized by abnormal sleep tendencies, including excessive daytime sleepiness and often disturbed nocturnal sleep and pathological manifestations of REM sleep ${ }^{1}$.We report a 12-year-old boy, who was initially treated as a case of epilepsy and later confirmed of having narcolepsy with cataplexy.

A 12-year-old boy presented with episodes of sudden fall, several times a day for last 6 months. These episodes of 5-10 seconds were associated with upward rolling of eyes. CT scan cranium and EEG were normal. A local practitioner made diagnosis of epilepsy and the child was put on phenytoin and sodium valproate. Even after 4 months of treatment, there was no reduction in symptoms and patient was referred to us. Detailed history revealed that child had a history of excessive daytime sleepiness for past one year. There was no history of sleep paralysis or hallucinations. Preceding emotions did not trigger these episodes of collapse. These events were not associated with change in posture, prolonged standing. There was no history of drug intake, palpitation, headache or anxiety. Anti-epileptic drugs were withdrawn and clinical diagnosis of narcolepsy with cataplexy was made. Overnight polysomnography was done and it was followed by multiple sleep latency test (MSLT) on next morning. Polysomnography was normal, but MSLT revealed sleep latency time of 0.7 minute and 2 episodes rapid eye movement (REM) sleep out of 4 recorded naps of 20 minute each. These findings of MSLT were consistent with narcolepsy. His parents were educated about the importance of scheduled naps, regular sleepwake schedule and overall good sleep hygiene. Modafinil acetamide $100 \mathrm{mg}$ two times a day was given for excessive daytime sleepiness. Clomipramine, $25 \mathrm{mg}$ at bedtime, was given for cataplexy. Clomipramine was withdrawn on subsequent visits. Presently the child is on modafinil alone for past 6 months and all symptoms have subsided.

Narcolepsy is often recognised by excessive daytime sleepiness and associated pathological manifestation of REM sleep like cataplexy, sleep paralysis and hypnagogic hallucinations. Automatic behaviours and disrupted nighttime sleep also occur commonly. Out of all REM sleep manifestations, cataplexy is unique to narcolepsy. It is an abrupt and reversible decrease or loss of muscle tone, most frequently elicited by emotion such as laughter, anger or surprise. Any preceding or anticipation of strong emotions did not trigger episodes of fall in our patient and there was no history of sleep paralysis, hallucination and disturbed sleep during night. Macleod $e t a l^{2}$ in a case series of five children reported preceding emotional factors in 4 children and 1 child had anticipation of strong emotional factor.

Diagnosis of narcolepsy requires a clinical history of sleepiness and a positive MSLT, with a mean sleep latency of less than 8 minute and 2 or more sleep-onset REM periods ${ }^{3}$.Our patient meet criteria of MSLT. Twenty-four hour video analysis did not record any event of collapse in our patient but we had observed 3 episodes of collapse during his visits.

Out of the 5 cases reported by Macleod et al ${ }^{2}, 1$ child was misdiagnosed as refractory epilepsy after few years of treatment on anti-epileptics drugs. Rest of 4 were suspected as a case of epilepsy. Modafinil is considered as drug of choice for excessive daytime sleepiness in narcolepsy $\mathrm{y}^{4}$. Behaviour approaches like scheduled naps, regular sleep-wake schedule, avoidance of frequent time zone changes and good sleep hygiene are very helpful.

Early diagnosis of narcolepsy is crucial for emotional, psychosocial and educational development of child. Narcolepsy/cataplexy should be included in differential diagnosis of paroxysmal disorders, particularly if there are associated sleep symptoms and behaviour difficulties. All patients of refractory epilepsy must be evaluated for sleep disorders especially cataplexy.

Anup Kumar Singh, Abhijeet Saha ${ }^{1}$, Naveen Dutt, P.R. Mohapatra, A.K. Janmeja and Anuradha Bansal ${ }^{1}$ Department of Pulmonary Medicine and ${ }^{1}$ Department of Pediatrics, Government Medical College \& Hospital, Chandigarh, India

E-mail :drabhijeetsaha@yahoo.com;

\section{REFERENCES}

1. Zeman A, Douglas N, Aylward R. Narcolepsy mistaken for epilepsy. BMJ 2001; 322 : 216-218.

2. Macleod S, Ferrie C, Zubberi SM. Symptoms of narcolepsy in children misinterpreted as epilepsy. Epileptic Disord 2005; 7: 13-17.

3. American Academy of Sleep Medicine. International Classification of Sleep Disorders. Diagnostic and Coding manual, $2^{\text {nd }}$ ed. Westchester, III, American Academy of Sleep Medicine, 2005

4. Bassetti C. Narcolepsy. Curr Treat Options Neurol 1999; 1: 291-298. 